\title{
Variation of Parameter Method for Solving Homogeneous Second Order Linear Ordinary Differential Equations
}

\section{Arficho D*}

Department of Mathematics, Aksum University, Aksum, Ethiopia

\begin{abstract}
In this manuscript, we introduce variation of parameter method for solving homogeneous linear second order ordinary differential equations with constant coefficients. Moreover, we derive general solution method to solve homogeneous linear second order ordinary differential equations with constant coefficients.
\end{abstract}

Keywords: Separable equation; Integration technique; Linear combination

\section{Introduction}

A differential equation is an equation that relates an unknown function and one or more of its derivatives of with respect to one or more independent variables [1]. If the unknown function depends only on a single independent variable, such a differential equation is ordinary differential equation. The order of an ordinary differential equation is the order of the highest derivative that appears in the equation [2]. A differential equation with an unknown function is said to be linear if it is linear in that unknown function and its derivatives. A differential equation of the form $a y^{(2)}+b y^{(1)}+c y=f(x)$ is said to be second order linear ordinary differential equation with constant coefficients if $\mathrm{a}, \mathrm{b}$ and $\mathrm{c}$ are constants and a is non zero scalar, where $f(x)$ is a function of $x$. The associated homogeneous equation of second order linear ordinary differential equation with constant coefficients $a y^{(2)}+b y^{(1)}+c y=f(x)$ is $a y^{(2)}+b y^{(1)}+c y=0$. First we solve equation $a y^{(2)}+b y^{(1)}+c y=0$ to solve second order linear ordinary differential equation with constant coefficients $a y^{(2)}+b y^{(1)}+c y=f(x)$. The polynomial $a p^{2}+b p+c$ is called the characteristic polynomial of $a y^{(2)}+b y^{(1)}+c y=0$ [3]. The function $y=\exp \int m d x$ is solution of $\mathrm{ay}^{(2)}+\mathrm{by}^{(1)}+\mathrm{cy}=0$ if and only if $\mathrm{m}$ is root of the characteristic polynomial $\mathrm{ap}^{2}+\mathrm{bp}+\mathrm{c}$ [3]. Thus, the solution of equation $a y^{(2)}+b y^{(1)}+c y=0$ is given by $y=\exp \int m d x$ whenever it is second order linear ordinary differential equation with constant coefficients, where $\mathrm{m}$ is a number such that $\mathrm{am}^{2}+\mathrm{bm}+\mathrm{c}=0$. We solve the characteristic equation $a m^{2}+b m+c=0$ of $a y^{(2)}+b y^{(1)}+c y=0$ to solve $a y^{(2)}+b y^{(1)}+c y=0$. Clearly the characteristic equation $\mathrm{am}^{(2)}+\mathrm{bm}+\mathrm{c}=0$ of $a \mathrm{y}^{(2)}+\mathrm{by}^{(1)}+\mathrm{cy}=0$ is quadratic equation in $\mathrm{m}$. Thus, we consider three cases for solution of the characteristic equation $\mathrm{am}^{2}+\mathrm{bm}+\mathrm{c}=0$ of $\mathrm{ay}^{(2)}+\mathrm{by}^{(1)}+\mathrm{cy}=0$. Most authors of differential books used this technique to derive solution method for solving second order linear ordinary differential equation with constant coefficients. We apply Moivre's theorem to find general solution of $a y^{(2)}+b y^{(1)}+c y=0$ when $b^{2}-4 a c<0$ [4]. In this manuscript, we introduce variation of parameter method to find general solution of homogeneous second order linear ordinary differential equation with constant coefficients. Furthermore, we do not use Moivre's theorem to find general solution of homogeneous second order linear ordinary differential equation with constant coefficients. If we replace a scalar $\mathrm{m}$ by a function $\mathrm{u}(\mathrm{x})$ and assume that $y=\exp \int u(x) d x$ is solution of homogeneous second order linear ordinary differential equation with constant coefficients, then $y=\exp \int u(x) d x$ is variation of parameter method for solving second order linear ordinary differential equation with constant coefficients, where $\mathrm{u}(\mathrm{x})$ is a function of $\mathrm{x}$ to be determined. Finally, we determine $\mathrm{u}(\mathrm{x})$ by assuming $y=\exp \int u(x) d x$ as solution of homogeneous second order linear ordinary differential equation with constant coefficients.

\section{Motivation Research Questions}

Determine $\mathrm{u}(\mathrm{x})$ if $y=\exp \int u(x) d x$ is solution of homogeneous second order linear ordinary differential equation with constant coefficients.

\section{Linear first order differential equations}

The linear first order ordinary differential equation with unknown dependent variable $\mathrm{y}$ and independent variable $\mathrm{x}$ is defined by

$$
a_{0}(x) y+a_{1}(x) y^{(1)}=g(x) .
$$

Solution of linear first order differential equations: The general solution of the equation in equation 1 is given by

$$
y=\frac{\int \frac{\mu(x) g(x)}{a_{1}} d x}{\mu(x)},
$$

where $\mu(x)=\exp \left(\int\left(\frac{a_{0}(x)}{a_{1}(x)}\right) d x\right)[5]$.

\section{Linear second order differential equations}

The second order linear ordinary differential equation with unknown dependent variable $\mathrm{y}$ and independent variable $\mathrm{x}$ is defined by

$$
a_{0}(x) y+a_{1}(x) y^{(1)}+a_{2}(x) y^{(2)}=g(x) .
$$

Fundamental set of solutions: A set of functions $\mathrm{y}_{1}(\mathrm{x}), \mathrm{y}_{2}(\mathrm{x}), \ldots, \mathrm{y}_{\mathrm{n}}(\mathrm{x})$ is said to be linearly dependent on an interval $I$ if there exist constants $\mathrm{c}_{1}, \mathrm{c}_{2}, \ldots \mathrm{c}_{\mathrm{n}}$ not all zero, such that $\mathrm{c}_{1} \mathrm{y}_{1}(\mathrm{x})+\mathrm{c}_{2} \mathrm{y}_{2}(\mathrm{x})+\ldots+\mathrm{c}_{\mathrm{n}} \mathrm{y}_{\mathrm{n}}(\mathrm{x})=0$ for every $\mathrm{x}$ in the interval. If the set of functions is not linearly dependent on the interval, it is said to be linearly independent. Any set $\mathrm{y}_{1}(\mathrm{x}), \mathrm{y}_{2}(\mathrm{x}), \ldots, \mathrm{y}_{\mathrm{n}}(\mathrm{x})$ of $\mathrm{n}$ linearly independent solutions of the homogeneous linear $n$ th-

${ }^{*}$ Corresponding author: Daniel Arficho, Department of Mathematics, Aksum University, Aksum, Ethiopia, Tel: +251910184808; E-mail: daniel.arficho@yahoo.com

Received August 16, 2015; Accepted September 24, 2015; Published September 29, 2015

Citation: Arficho D (2015) Variation of Parameter Method for Solving Homogeneous Second Order Linear Ordinary Differential Equations. J Appl Computat Math 4 258. doi:10.4172/2168-9679.1000258

Copyright: ( 2015 Arficho D. This is an open-access article distributed under the terms of the Creative Commons Attribution License, which permits unrestricted use, distribution, and reproduction in any medium, provided the original author and source are credited. 
order differential equation on an interval $\mathrm{I}$ is said to be a fundamental set of solutions on the interval [3].

General solution of linear second order differential equations: The associated homogeneous differential equation of nonhomogeneous linear nth order differential equation

$a_{0}(x) y+a_{1}(x) y^{(1)}+\cdots+a_{n-1}(x) y^{(n-1)}+a_{n}(x) y^{(n)}=g(x)$

is

$a_{0}(x) y+a_{1}(x) y^{(1)}+\cdots+a_{n-1}(x) y^{(n-1)}+a_{n}(x) y^{(n)}=0$ Dennis

Theorem 1: Let $\mathrm{y}_{1}, \mathrm{y}_{2}, \ldots, \mathrm{y}_{\mathrm{n}}$ be linearly independent solutions of the homogeneous linear nth order differential equation

$a_{0}(x) y+a_{1}(x) y^{(1)}+\cdots+a_{n-1}(x) y^{(n-1)}+a_{n}(x) y^{(n)}=0$

on an interval I. Then the general solution of the equation in 4 on the interval I is

$y=c_{1} y_{1}+c_{2} y_{2}+\cdots+c_{n-1} y_{n-1}+c_{n} y_{n}$,

Where $c_{i},(i=1,2, \ldots, n-1, n)$ are arbitrary constants $[6]$.

The general solution of the equation in equation 3 is

$y=c_{1} y_{1}+c_{2} y_{2}+y_{p}$,

Where $y_{1}$ and $y_{2}$ are linearly independent solutions of the associated homogeneous equation of the equation in 3 and $y_{p}$ is particular solution of the equation in 3 . Here $c_{i},(i=1,2)$ are arbitrary constants [5].

\section{Construction of a second solution from a known solution: Let}

$$
a y^{(2)}+b y^{(1)}+c y=o
$$

be homogeneous linear second order ordinary differential equation with constant coefficients. Let $y_{1}$ be a non-zero known solution of the equation in 6 . Thus, we have

$$
c y_{1}+b y_{1}^{(1)}+a y_{1}^{(2)}=0
$$

Then we assume that $\mathrm{y}_{2}(\mathrm{x})=\mathrm{u}(\mathrm{x}) \mathrm{y}_{1}(\mathrm{x})$ as another solution of the equation in equation 6 to construct the second solution of the equation in equation 6 .

It follows that $y_{2}^{(1)}(x)=u^{(1)}(x) y_{1}(x)+u(x) y_{1}^{(1)}(x) \quad$ and $y_{2}^{(2)}(x)=u^{(2)}(x) y_{1}(x)+2 u^{(1)}(x) y_{1}^{(1)}(x)+u(x) y_{1}^{(2)}(x)$. Since $y_{2}(x)=u(x) y_{1}(x)$ is solution of the equation in equation 6 , we have

$$
c y_{2}+b y_{2}^{(1)}+a y_{2}^{(2)}=0
$$

From equation in equation 8 it follows that

$$
c u(x) y_{1}(x)+b\left[u(x) y_{1}^{(1)}+u^{(1)} y_{1}\right]+a\left[u(x) y_{1}^{(2)}+2 y_{1}^{(1)} u^{(1)}+u^{(2)} y_{1}\right]=0 \text {. }
$$

Using equation in equation 7 , the equation in equation 9 is reduced to

$$
b\left[u^{(1)} y_{1}\right]+a\left[2 y_{1}^{(1)} u^{(1)}+u^{(2)} y_{1}\right]=0
$$

From equation in equation 10 it follows that

$$
\left[b y_{1}+2 a y_{1}^{(1)}\right] u^{(1)}+\left[a y_{1}\right] u^{(2)}=0
$$

Let $\mathrm{u}^{(1)}=\mathrm{V}$. Then from equation in equation 11 , we have

$$
\left[b y_{1}+2 a y_{1}^{(1)}\right] v+\left[a y_{1}\right] v^{(1)}=0
$$

The equation in equation 12 is linear first order ordinary differential equation. Thus, using the formula in equation 2 , we get

$$
v=\frac{\int \frac{\mu(x) 0}{a y_{1}} d x}{\mu(x)}=\frac{1}{\mu(x)},
$$

where $\mu(x)=\exp \left(\int\left(\frac{b y_{1}+2 a y_{1}^{(1)}}{a y_{1}}\right) d x\right)=y_{1}^{2} \exp \left(\int\left(\frac{b}{a}\right) d x\right)$.

Thus, $u=\int v d x$, where

$$
v=\frac{1}{\mu(x)} \text { and } \mu(x)=y_{1}^{2} \exp \left(\int\left(\frac{b}{a}\right) d x\right) .
$$

Therefore, the second solution $\mathrm{y}_{2}(\mathrm{x})=\mathrm{u}(\mathrm{x}) \mathrm{y}_{1}(\mathrm{x})$ of the equation in equation 6 is

$y_{2}(x)=y_{1}(x) \int v d x$,

where

$\nu=\frac{1}{\mu(x)}$ and $\mu(x)=y_{1}^{2} \exp \left(\int\left(\frac{b}{a}\right) d x\right)$.

From Theorem 1 we observe that

$y=c_{1} y_{1}+c_{2} y_{2}$,

is the general solution of 6 , where $y_{1}$ and $y_{2}$ are linearly independent solutions of the equation in 6 .

The general solution of the equation in equation 3 is

$$
y=c_{1} y_{1}+c_{2} y_{2}+y_{p},
$$

Where $y_{1}$ and $y_{2}$ are linearly independent solutions of the associated homogeneous equation of the equation in 3 and $y_{p}$ is particular solution of the equation in 3 . Here $c_{i},(i=1,2)$ are arbitrary constants [5].

\section{General Solution of Homogeneous Second Order Linear Ordinary Differential Equation with Constant Coefficients}

Theorem 2: The general solution of second order linear ordinary differential equation $a y^{(2)}+b y^{(1)}+c y=0$ with constant coefficients $\mathrm{a}, \mathrm{b}$ and $\mathrm{c}$ is $y=c_{1} x \exp \alpha x+c_{2} \exp \alpha x$ if $4 a c-b^{2}=0$ and $\alpha=\frac{-b}{2 a}$ $y=\exp \alpha x\left[c_{1} \cos \beta x+\sin \beta x\right]$ if $4 a c-b^{2}>0, \alpha=\frac{-b}{2 a}$ and $\beta=\frac{\sqrt{4 a c-b^{2}}}{2 a}$ $y=c_{1} \exp (\alpha+\beta) x+\exp (\alpha-\beta) x$ if $4 a c-b^{2}<0, \alpha=\frac{-b}{2 a}$ and $\beta=\frac{\sqrt{b^{2}-4 a c}}{2 a}$

Proof: We know that most authors assumed that $y=\exp \int m d x$ as solution of homogeneous second order linear ordinary differential equations with constant coefficients, where $\mathrm{m}$ is a scalar to be determined. In this manuscript, We introduce variation of parameter method to prove this theorem. First we assume that $y=\exp \int u(x) d x$ as solution of homogeneous second order linear ordinary differential equations with constant coefficients, where $\mathrm{u}(\mathrm{x})$ is a function to be determined. Then we transform a homogeneous second order linear ordinary differential equation with constant coefficients to separable first order ordinary differential equation by this assumption. Finally, we solve an equivalent separable first order ordinary differential equation to prove this theorem.

Suppose that $y=\exp \int u(x) d x$ is solution of the equation in 6 . It follows that $\mathrm{y}_{(1)}=$ uy and $\mathrm{y}^{(2)}=\left(\mathrm{u}^{(1)}+\mathrm{u}_{2}\right) \mathrm{y}$.

Since $y=\exp \int u(x) d x$ is solution of the equation in 6, we have $a\left(u^{(1)}+u^{2}\right) y+b u y+c y=o$. Since $y$ is non zero function, it follows that

$a\left(u^{(1)}+u^{2}\right)+b u+c=o$ 
Consider the expression $a\left(u^{(1)}+u^{2}\right)+b u+c$.

$$
\begin{aligned}
& a\left(u^{(1)}+u^{2}\right)+b u+c=a u^{(1)}+a u^{2}+b u+c \\
& =a u^{(1)}+a\left[u^{2}+\frac{b}{a} u+\frac{c}{a}\right] \\
& =a u^{(1)}+a\left[u^{2}+\frac{b}{a} u+\frac{b^{2}}{4 a^{2}}+\frac{c}{a}-\frac{b^{2}}{4 a^{2}}\right] \\
& =a u^{(1)}+a\left[\left(u+\frac{b}{2 a}\right)^{2}+\frac{4 a c-b^{2}}{4 a^{2}}\right] \\
& =a u^{(1)}+a\left[\left(u+\frac{b}{2 a}\right)^{2}\right]+\frac{4 a c-b^{2}}{4 a}
\end{aligned}
$$

Thus,

$a\left(u^{(1)}+u^{2}\right)+b u+c=a u^{(1)}+a\left[\left(u+\frac{b}{2 a}\right)^{2}\right]+\frac{4 a c-b^{2}}{4 a}$

Let $u+\frac{b}{2 a}=v$. Then we have $\mathrm{u}^{(1)}=\mathrm{v}^{(1)}$. Thus, the equation in 17 is equivalent to $a v^{(1)}+a v^{2}+\frac{4 a c-b^{2}}{4 a}=0$.

Hence

$$
v^{(1)}+v^{2}+\frac{4 a c-b^{2}}{4 a^{2}}=0
$$

The equation in 19 is separable first order ordinary differential equation because it is equivalent to

$$
-d x=\frac{d v}{\left(v^{2}+\frac{4 a c-b^{2}}{4 a^{2}}\right)}
$$

We consider three cases to solve the equation in 20 .

Case I: Suppose that $4 a c-b^{2}=0$. Then the equation in 20 is equivalent to $-d x=\frac{d v}{v^{2}}$. This implies that $v=\frac{1}{x}$.

Let $\alpha=\frac{-b}{2 a}$. Then $u=\frac{1}{x}+\alpha$.

Thus, $y=\exp \int u(x) d x=\exp \int\left(\frac{1}{x}+\alpha\right) d x=x \exp \int \alpha d x=x \exp \left(\frac{-b}{2 a}\right) x$

Therefore, $y_{1}=x \exp \left(\frac{-b}{2 a}\right) x$ is solution of the equation in 6 .

We construct the second solution of the equation in 6 by using the formula in equation 14. Thus, $\mu(x)=y_{1}^{2} \exp \left(\int\left(\frac{b}{a}\right) d x\right)=x^{2}$ and $v=\frac{1}{x^{2}}$ Hence

$$
y_{2}(x)=y_{1}(x) \int \frac{1}{x^{2}} d x=\frac{-1}{x}\left(x \exp \left(\frac{-b}{2 a}\right) x\right)=-\exp \left(\frac{-b}{2 a}\right) x
$$

Therefore, $y=c_{1} x \exp \alpha x+c_{2} \exp \alpha x$ is general solution of the equation in 6 because $y$ is linear combination of $y_{1}$ and $y_{2}$ if $4 a c-b^{2}=0$ and $\alpha=\frac{-b}{2 a}$

Case II: Suppose that $4 \mathrm{ac}-\mathrm{b}^{2}>0$. Let $\alpha=\frac{-b}{2 a}$ and $\beta=\frac{\sqrt{4 a c-b^{2}}}{2 a}$. Then the equation in 20 is equivalent to $-d x=\frac{d v}{\beta^{2}+v^{2}}$. This implies that $-x=\frac{1}{\beta} \arctan \left(\frac{v}{\beta}\right)$.

It follows that $\frac{v}{\beta}=\tan (-\beta x)=-\tan (\beta x)$. This implies that $v=-\beta \tan (\beta x)$. Since $\mathrm{u}=\mathrm{v}+\alpha$, we have $u=-\beta \tan (\beta x)+\alpha$. It follows that $\int u(x) d x=\int(-\beta \tan (\beta x)+\alpha) d x=\alpha x+\ln (\cos \beta x)$.
Therefore, $y_{1}=(\cos \beta x) \exp \alpha x$ is solution of the equation in 6 whenever $4 \mathrm{ac}^{\mathrm{c}} \mathrm{b}^{2}>0, \quad \alpha=\frac{-b}{2 a}$ and $\beta=\frac{\sqrt{4 a c-b^{2}}}{2 a}$. From this known solution we can construct the second solution by using equation in 14 . $u=\int\left(\frac{\exp 2 \alpha x}{\exp 2 \alpha x \cos ^{2} x}\right) d x=\int \sec ^{2} x d x=\frac{\tan (\beta x)}{\beta}$

Thus,

$y_{2}(x)=y_{1}(x) u(x)=(\cos \beta x)(\exp \alpha x)\left(\frac{\tan (\beta x)}{\beta}\right)=\frac{1}{\beta}(\sin \beta x)(\exp \alpha x)$

Therefore, $y=\exp \alpha x\left[c_{1} \cos \beta x+\sin \beta x\right]$ is general solution of the equation in 6 because $y$ is linear combination of $y_{1}$ and $y_{2}$ if $4 a c-b^{2}>0$, $\alpha=\frac{-b}{2 a}$ and $\beta=\frac{\sqrt{4 a c-b^{2}}}{2 a}$.

Case III: Suppose that $4 \mathrm{ac}-\mathrm{b}^{2}<0$. Let $\alpha=\frac{-b}{2 a}$ and $\beta=\frac{\sqrt{b^{2}-4 a c}}{2 a}$. Then the equation in 20 is equivalent to $d x=\frac{d v}{\beta^{2}-v^{2}}$.

Consider $\int \frac{1}{\beta^{2}-v^{2}} d v$.

$\int \frac{1}{\beta^{2}-v^{2}} d v=\int \frac{1}{(\beta-v)(\beta+v)} d v$

$=\frac{1}{2 \beta} \int\left(\frac{1}{\beta-v}+\frac{1}{\beta+v}\right) d v \quad=\frac{1}{2 \beta} \int \frac{1}{\beta-v} d v+\frac{1}{2 \beta} \int \frac{1}{\beta+v} d v$

$=\frac{1}{2 \beta}[\ln (\beta+v)-\ln (\beta-v)]$

$=\frac{1}{2 \beta}\left[\ln \left(\frac{\beta+v}{\beta-v}\right)\right]$

This implies that $x=\frac{1}{2 \beta}\left[\ln \left(\frac{\beta+v}{\beta-v}\right)\right]$.

It follows that $\frac{\beta+v}{\beta-v}=\exp 2 \beta x$.

This implies that $v(1+\exp 2 \beta x)=\beta(\exp 2 \beta x-1)$.

Thus, $v=\beta\left[\frac{\exp 2 \beta x-1}{1+\exp 2 \beta x}\right]$.

Since $\mathrm{u}=\mathrm{v}+\alpha$, we have $u=\beta\left[\frac{\exp 2 \beta x-1}{1+\exp 2 \beta x}\right]+\alpha$.

It follows that

$$
\begin{aligned}
\int u(x) d x & =\int\left(\beta\left(\frac{\exp 2 \beta x-1}{1+\exp 2 \beta x}\right)+\alpha\right) d x \\
& \left.=\int \beta\left(\frac{\exp 2 \beta x-1}{1+\exp 2 \beta x}\right) d x+\int \alpha\right) d x \\
& =\beta \int\left(\frac{\exp 2 \beta x-1}{1+\exp 2 \beta x}\right) d x+\alpha x \\
& =\beta \int\left(\frac{\exp 2 \beta x+1-2}{1+\exp 2 \beta x}\right) d x+\alpha x \\
& =\beta \int\left(1-2 \frac{1}{1+\exp 2 \beta x}\right) d x+\alpha x \\
= & \beta x-2 \beta \int \frac{1}{1+\exp 2 \beta x} d x+\alpha x
\end{aligned}
$$

Thus, 


$$
\int u(x) d x=\beta x-2 \beta \int \frac{1}{1+\exp 2 \beta x} d x+\alpha x
$$

Let's evaluate $\int \frac{1}{1+\exp 2 \beta x} d x$. Let $z=1+\exp 2 \beta x$. Then $d z=2 \beta \exp 2 \beta x d x$

This implies that $d x=\frac{\exp -2 \beta x}{2 \beta} d z=\frac{1}{(2 \beta)(z-1)} d z$. Thus,

$$
\begin{gathered}
\int \frac{1}{1+\exp 2 \beta x} d x=\frac{1}{2 \beta} \int \frac{1}{z(z-1)} d z \\
=\frac{1}{2 \beta} \int \frac{1}{z-1}-\frac{1}{z} d z \\
=\frac{1}{2 \beta}[\ln (z-1)-\ln (z)] \\
=\frac{1}{2 \beta} \ln \left(\frac{z-1}{z}\right) \\
=\frac{1}{2 \beta} \ln \left(\frac{\exp 2 \beta x}{1+\exp 2 \beta x}\right)
\end{gathered}
$$

Thus,

$$
\int \frac{1}{1+\exp 2 \beta x} d x=\frac{1}{2 \beta} \ln \left(\frac{\exp 2 \beta x}{1+\exp 2 \beta x}\right)
$$

It follows from equations in 23 and 24 that

$$
\int u(x) d x=(\alpha+\beta) x-\ln \left(\frac{\exp 2 \beta x}{1+\exp 2 \beta x}\right)
$$

Thus, $y_{1}=\left(\frac{1+\exp 2 \beta x}{\exp 2 \beta x}\right) \exp (\alpha+\beta) x=(1+\exp 2 \beta x) \exp (\alpha-\beta) x$

$$
=\exp (\alpha+\beta) x+\exp (\alpha-\beta) x
$$

Therefore, $y_{1}=\exp (\alpha+\beta) x+\exp (\alpha-\beta) x$ is solution of the equation in 6 whenever $4 \mathrm{ac}-\mathrm{b}^{2}<0, \alpha=\frac{-b}{2 a}$ and $\beta=\frac{\sqrt{b^{2}-4 a c}}{2 a}$. From this known solution we can construct the second solution by using equation in 14 . Let's consider u in equation 14.

$$
\begin{aligned}
& u=\int\left(\frac{\exp 2 \alpha x}{(\exp (\alpha+\beta) x+\exp (\alpha-\beta) x)^{2}}\right) d x \\
& =\int\left(\frac{1}{(\exp \beta x+\exp -\beta x)^{2}}\right) d x \\
& =\int\left(\frac{\exp 2 \beta x}{(1+\exp 2 \beta x)^{2}}\right) d x
\end{aligned}
$$

Thus,

$$
u=\int\left(\frac{\exp 2 \alpha x}{(\exp (\alpha+\beta) x+\exp (\alpha-\beta) x)^{2}}\right) d x=\int\left(\frac{\exp 2 \beta x}{(1+\exp 2 \beta x)^{2}}\right) d x
$$

Let $z=1+\exp 2 \beta x$. Then $d z=2 \beta \exp 2 \beta x d x$.

This implies that $\exp 2 \beta x d x=\frac{1}{2 \beta} d z$.

$$
\begin{aligned}
& \int\left(\frac{\exp 2 \beta x}{(1+\exp 2 \beta x)^{2}}\right) d x=\frac{1}{2 \beta} \int \frac{1}{z^{2}} d z \\
& =-\frac{1}{2 \beta z}
\end{aligned}
$$

$$
\begin{aligned}
& =-\frac{1}{2 \beta(1+\exp 2 \beta x)} \\
& =\left(-\frac{1}{2 \beta}\right) \frac{\exp -\beta x}{(\exp \beta x+\exp -\beta x)}
\end{aligned}
$$

Thus,

$$
\int\left(\frac{\exp 2 \beta x}{(1+\exp 2 \beta x)^{2}}\right) d x=\left(-\frac{1}{2 \beta}\right) \frac{\exp -\beta x}{(\exp \beta x+\exp -\beta x)}
$$

It follows from equations in 26 and 27 that

$$
u=\int\left(\frac{\exp 2 \alpha x}{(\exp (\alpha+\beta) x+\exp (\alpha-\beta) x)^{2}}\right) d x=\left(-\frac{1}{2 \beta}\right) \frac{\exp -\beta x}{(\exp \beta x+\exp -\beta x)}
$$

Thus,

$$
y_{2}(x)=y_{1}(x) u=y_{1}(x)\left[\left(-\frac{1}{2 \beta}\right) \frac{\exp -\beta x}{(\exp \beta x+\exp -\beta x)}\right]=\left(-\frac{1}{2 \beta}\right) \exp (\alpha-\beta) x
$$

Therefore, $y=c_{1} \exp (\alpha+\beta) x+\exp (\alpha-\beta) x$ is general solution of the equation in 6 because $\mathrm{y}$ is linear combination of $\mathrm{y}_{1}$ and $\mathrm{y}_{2}$ if $4 \mathrm{ac}-\mathrm{b}^{2}<0$, $\alpha=\frac{-b}{2 a}$ and $\beta=\frac{\sqrt{b^{2}-4 a c}}{2 a}$.

Hence proved

\section{Results and Discussion}

Most authors of differential equations used Moivre's theorem to find general solution of $a y^{(2)}+b y^{(1)}+c y=0$ when $b^{2}-4 a c<0$ [4]. In this manuscript, we introduced variation of parameter method to find general solution of homogeneous second order linear ordinary differential equation with constant coefficients. Furthermore, we found general solution of homogeneous second order linear ordinary differential equation with constant coefficients without applying Moivre's theorem .

\section{Conclusion}

In this manuscript, we determined undetermined functions $\mathrm{u}(\mathrm{x})$ by assuming $y=\exp \int u(x) d x$ as solution of homogeneous second order linear ordinary differential equation with constant coefficients. Moreover, we proved in theorem 2 by applying variation of parameter method for solving homogeneous second order linear ordinary differential equation with constant coefficients.

\section{References}

1. Dennis GZ (2013) A First Course in Differential Equations. Ricard Stratton, Los Angeles, United States of America.

2. Yuksel S (2014) Differential Equations for Engineering Science. Queen's University, Canada.

3. Ogunrinde RB, Sunday J (2012) On Some Models Based on Second Order Differential Equations. Am J Sci Ind Res 3: 288-291.

4. Gonze D, Abou-Jaoude W (2012) Linear differential Equations.

5. Arficho D (2015) Method for Solving Particular Solution of Linear Second Order Ordinary Differential Equations. J Appl Computat Math 4: 210.

6. Levermore D (2012) Higher-Order Linear Ordinary Differential Equations I: Introduction and Homogeneous Equations. Department of Mathematics, University of Maryland 